\title{
Uncertainty Quantification for Turbulent Mixing Flows: Rayleigh-Taylor Instability
}

\author{
T. Kaman ${ }^{1}$, R. Kaufman ${ }^{1}$, J. Glimm ${ }^{1}$, and D.H. Sharp ${ }^{2}$ \\ 1 Department of Applied Mathematics and Statistics \\ Stony Brook University \\ Stony Brook, NY USA \\ 2 Los Alamos National Laboratory \\ Los Alamos, NM, USA
}

\begin{abstract}
Uncertainty Quantification (UQ) for fluid mixing depends on the length scales for observation: macro, meso and micro, each with its own UQ requirements. New results are presented here for macro and micro observables. For the micro observables, recent theories argue that convergence of numerical simulations in Large Eddy Simulations (LES) should be governed by space-time dependent probability distribution functions (PDFs, in the present context, Young measures) which satisfy the Euler equation. From a single deterministic simulation in the LES, or inertial regime, we extract a PDF by binning results from a space time neighborhood of the convergence point. The binned state values constitute a discrete set of solution values which define an approximate PDF. The convergence of the associated cumulative distribution functions (CDFs) are assessed by standard function space metrics.
\end{abstract}

\section{Introduction}

LES convergence is an asymptotic description of numerical simulations of the inertial, or self similar, scaling range of a turbulent flow. In the LES regime we are not concerned with convergence in a conventional sense. Such mathematical convergence to a classical or weak solution, as $\Delta x \rightarrow 0$, is a property of direct numerical simulations (DNS), i.e., simulations with all length scales resolved. For practical problems of turbulence this goal may be unrealistic. By contrast, in the following we investigate LES convergence, defined as the behavior of numerical solutions in the LES (inertial) regime under mesh refinement. In this regime there is still a type of convergence but it may be weaker than that considered by traditional DNS analysis. For example, rather than convergence to a weak solution, it may be useful or even necessary to consider convergence of probability distribution functions (PDFs) to a measure valued solution (Young measure). The PDFs capture the local fluctuations of the solution, which are an important aspect of the solution in the inertial regime.

In this article, we present such a picture, still incomplete, from perspectives of mathematical theory, simulation and physical reasoning. It allows these two notions of convergence (DNS and LES; classical and $\mathrm{w}^{*}$ Young measure limit) to coexist. 
The main result is a convergence study for PDFs and CDFs for a numerical mesh refinement study of a Rayleigh-Taylor problem. At present meshes, we find CDF but not yet PDF convergence, a minimum sampling size (supercell size) for the stochastic convergence, and suitable norms for the measurement of convergence.

\section{Verification, Validation and Uncertainty Quantification for RT Mixing}

\subsection{The RT Mixing Rate $\alpha$}

The Rayleigh-Taylor (RT) instability is a classical hydrodynamical instability driven by an acceleration force applied across a density discontinuity. The result is a mixing layer, growing in time with a penetration thickness (of the bubbles, i.e. the light fluid)

$$
h_{b}=\alpha_{b} A g t^{2}
$$

where $A$ is the Atwood number, $\alpha_{b}$ is a dimensionless buoyancy correction factor, and $g$ is the acceleration force. We have achieved excellent agreement with experiment in our RT simulations; see Table 1 The results of Table 1, being stronger than LES simulations of others, require detailed examination. A distinctive algorithmic feature of our simulations is the combined use of front tracking and subgrid scale models for LES, or FT/LES/SGS in brief. A second feature of our work has been careful modeling of experimental detail. We summarize here two issues important to this examination: initial conditions and mesh resolution.

\subsection{Uncertainty Quantification for Initial Conditions and Mesh Convergence}

For most experiments, the initial conditions were not recorded, and the possibility of influence of long wave length initial perturbations has been a subject of speculation. We have quantified the allowed long wave length perturbation amplitudes, by an analysis of the recorded early time data [564]. Including an

Table 1. Comparison of FT/LES/SGS simulation to experiment. Simulation and experimental results reported with two significant digits. Discrepancy refers to the comparison of results outside of uncertainty intervals, if any, as reported.

\begin{tabular}{|c|c|c|c|c|c|}
\hline Ref. & Exp. & Sim. Ref. & $\alpha_{\exp }$ & $\alpha_{\mathrm{sim}}$ & Discrepancy \\
\hline 21 & \#112 & 8 & 0.052 & 0.055 & $6 \%$ \\
\hline 21 & \#105 & 4 & 0.072 & $0.076 \pm 0.004$ & $0 \%$ \\
\hline 2120 & 10 exp. & 3 & $0.055-0.077$ & 0.066 & $0 \%$ \\
\hline 19 & air-He & 13 & $0.065-0.07$ & 0.069 & $0 \%$ \\
\hline 17 & Hot-cold & 84 & $0.070 \pm 0.011$ & 0.075 & $0 \%$ \\
\hline 17 & Salt-fresh & 4 & $0.085 \pm 0.005$ & 0.084 & $0 \%$ \\
\hline
\end{tabular}




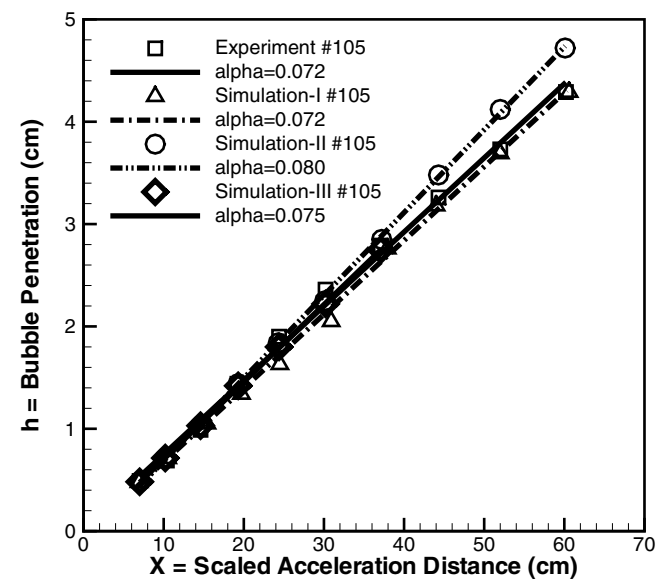

Fig. 1. Plot of the bubble penetration distance $h_{b}$ vs. a scaled acceleration distance $A g t^{2}$. The slope is the mixing growth rate $\alpha_{b}$. We plot the experimental data points and three simulation results, which have (I) $0 \times$ and (II) $2 \times$ our best reconstruction of the initial long wave length perturbations, as extrapolated by backward propagation in time from the early time experimental plates. (III) Inferred initial conditions for long wave length perturbations fully resolved, with a mesh $\Delta x=111 \mu \mathrm{m}<l_{\mathrm{We}}=780 \mu \mathrm{m}$ where $l_{\text {We }}$ is the critical bubble size (predicted by Weber number theory). The simulation III is still in progress.

estimate of the uncertainty of this backward extrapolation of data propagated backward to $t=0$, we estimate the uncertainty in $\alpha_{b}$ to be $10 \%$ or less, based on simulations which included (I) no initial long wave length perturbation and (II) double the reconstructed long wave length perturbation amplitudes. This range of initial conditions encompasses our estimates in the uncertainty of the reconstruction. See Figure 1 .

\section{Young Measures}

We explain the concept of a Young measure. For a turbulent flow, in the inertial regime, i.e, for LES simulations of turbulence, the Young measure description of the flow is a much deeper and more useful notion than is a classical weak solution or its numerical approximation. We generalize the notion of test function and of observation, using expectation values $\langle\cdots\rangle$ defined for the integration over the (state) random variables. See also 9 .

To start, we suppress the spatial dependence. Thus we have a random system, whose state $\xi$ takes on random values. We introduce a measure space $\Omega$ with $\xi \in \Omega$ and a probability measure (unit total measure) $d \nu(\xi)$ on $\Omega$. We denote the result of integrating with respect to $\nu$ as $\langle\cdots\rangle$. Then $\langle 1\rangle=\int_{\Omega} d \nu(\xi)=1$.

A measurement is defined by a continuous function $f$ of $\xi$, and defines a mean or expected value of repeated measurements of $f$ in the random system state 
$d \nu$, given by the integral

$$
\langle f\rangle=\int_{\Omega} f(\xi) d \nu(\xi)
$$

If the expectation yields the value $1 / 2$, we may conclude that repeated measurements will give a $50 \%$ measurement for $f$, on the average. But we do not know whether the value $1 / 2$ occurs with each measurement (probability 1 ), i.e., perfect mixing with no fluctuations, or whether, at the other extreme, the value 1 occurs with probability $1 / 2$, that is, no mixing at all and total fluctuations. For further information, we look at moments. The second moment of the concentration $(f(\xi)=\xi)$, useful for chemical reaction kinetics, is

$$
\langle f(1-f)\rangle=\int_{\Omega} f(\xi)(1-f(\xi)) d \nu(\xi) .
$$

Eq. (3) gives information regarding the spread, or dispersion, of the measure $\nu$. A common normalization of (3), is the coefficient of variation for $f$,

$$
\theta=\frac{\langle f(1-f)\rangle}{\langle f\rangle\langle(1-f)\rangle} .
$$

Now we add a spatial and temporal variability to all of the above. The measure $d \nu_{x, t}(\xi)$ now depends on $x, t$. The added value in allowing such a Young measure as a solution is that the local fluctuations are intrinsically associated with the space time point $x, t$.

The measurement defined by the stochastic observable $g(x, t, \xi)$ yields the expected value $\langle g(x, t, \cdot)\rangle$ at the space time point $x, t$. We expect this function of $x, t$ to be a distribution, and so assuming that $g$ is smooth (a test function) in its dependence on $x, t$, the outcome of the measurement is $\int\langle g\rangle d x d t$. Through this formalism, we can apply differential operators to the state $d \nu$, and as we have a governing PDE, we require $d \nu$ to be a solution of this PDE.

In contrast to multiplication by a test function for a weak solution, the values of the $\mathrm{w}^{*}$ limit test function $g$ multiply probabilities, while the state variable values (density, momentum, concentration), etc., the usual units for the values of the test function, now show up as an argument $\xi$ of $g$. See Table 2 .

A natural role for Young measures in a mathematical theory of the Euler equation and their relation to the Kolmogorov turbulence theory is discussed in [1]

Table 2. Comparison of weak solutions and Young measures in terms of test functions

\begin{tabular}{|l|l|l|}
\hline & weak solutions & Young measures \\
\hline$g$ values multiply & state variables & probabilities \\
$g$ arguments & space, time $x, t$ & space, time, $x, t ;$ state values $\xi$ \\
integration domain & space-time & space-time; state values \\
example & $g(x, t)$ multiplies & $g(x, t, \xi)$ multiplies \\
& momentum, energy, concentration & probability \\
\hline
\end{tabular}


and references cited there. In this reference we assume bounds from Kolmogorov theory, which serve as a type of Sobelov inequality for the approximations, and derive strong convergence for solutions of the incompressible Navier-Stokes equations (after passage to a subsequence) to weak solutions of the Euler equation limit, and $w^{*}$ convergence for passive scalars coupled to the Navier-Stokes velocity field, to an Euler equation Young measure limit.

\section{Verification for Stochastic (Young Measure) Convergence}

The point of view presented here $-w^{*}$ convergence to a Young measure solution and the coarse grain and sample algorithm to support this type of convergence numerically - needs verification and validation. Preliminary results in this direction have been established [1110127]. To discuss convergence of measures, we need to introduce function spaces for convergence. The PDFs themselves are noisy, and convergence of the PDFs directly appear to require difficult levels of mesh resolution. We introduced [10] for this purpose the indefinite integral of the PDFs, namely the probability distribution functions, i.e., the cumulative distribution functions (CDFs). These are better behaved and easier to analyze. Standard function space norms on the CDFs can be used, such as $L_{1}$ or the Kolmogorov-Smirnov norm $L_{\infty}$.

We study nonlinear functions of the solution through analysis of second moments. The convergence properties of the second moments depend on the specific variables which enter into the second moment; some converge nicely while others would benefit from a larger statistical ensemble and/or further mesh refinement.

$W^{*}$ convergence assumes an integration both over the solution state variables and over space and time. It applies to nonlinear functions of the solution. The idea of stochastic convergence is naturally appealing to workers versed in turbulence modeling. It is, however, a point of view which has not had extensive study in the numerical analysis literature, probably due to the requirements or perceived requirements for mesh resolution and the known limits of practicality for DNS simulations of many realistic problems. For this reason, it is of considerable interest to document exactly what is needed to achieve exactly which levels of convergence in exactly which topology.

Here we investigate multiple realizations of these ideas, in that the tradeoffs and issues related to stochastic convergence appear not to be well documented in the numerical analysis literature. We study integrated convergence through an $L_{1}$ norm (relative to integration both in solution state variables and over space-time) for the CDFs. We see that the $L_{1}$ norm for spatial integration is preferred to an $L_{\infty}$ norm, and that this choice for the CDFs appears to be showing convergence. Additional mesh refinement, which we anticipate in the future as a result of increased computing power, will clarify this property.

We also explore the size of the supercell used to define the PDFs and CDFs. This size defines a tradeoff between enhanced statistical convergence and the quality of the mesh (supercell mesh) resolution. The $L_{1}$ norm convergence is 
enhanced with larger supercells. We study convergence of the PDFs directly. The PDFs do not show convergence in the $L_{1}$ norm with present levels of numerical and statistical resolution, but the trend of results suggests that convergence is possible with further mesh refinement.

\subsection{Convergence of Second Moments}

Here we show the convergence under mesh refinement of the second moments for species concentration and velocities, two quantities of interest in a miscible Rayleigh-Taylor experiment 21. Since the quantities we report were not measured experimentally, this study is verification only, not validation. A related simulation study [9] includes comparison to the water channel experiments [15]16, in which the second moments were measured, and thus for which validation was studied.

It is commonly believed (and observed in numerical studies) that fluctuating quantities obey a type of Kolmogorov scaling law. This property, if correct, implies that the fluctuations are represented by a convergent integral, and should exhibit convergence under mesh refinement. Thus the convergence we report here should not be a surprise. Still, our results provide new information with respect to the level of refinement needed to observe convergent behavior. We generally observe satisfactory convergence through comparison between the medium and finest of the three spatial grids considered here, and unsatisfactory (poor agreement with the refined grid) properties for the coarsest grid. The limits at late time encounter a varying loss of statistical resolution due to the diminished number of statistically independent degrees of freedom at late time. The three grids have a size 520 to 130 microns ( 4 to 8 to 16 cells per elementary initial wave length). Of these, we have generally used the medium grid in our previous simulations, while the coarse grid is commonly favored in RT studies [2]. All second moments reported here represent mid plane values, i.e. a slice $z=$ const from the center of the mixing zone with $t$ fixed, and are averaged over all $x, y$ values.

The second moments of concentration, normalized to define the molecular mixing correlation $\theta=\langle f(1-f)\rangle /\langle f\rangle\langle 1-f\rangle$, were studied experimentally (distinct experiments, not reviewed here). Our value for $\theta \approx 0.8$ is consistent with values obtained numerically in related problems by others. However, significantly smaller $\theta$ values were observed in the similar fresh-salt water miscible experiments [1516. Since these differences are observed even at very early times, we can attribute the differences to initial conditions, specifically to the thickness of the initial diffusion layer. Fig. 2 displays numerical results for convergence of $\theta$ which model experiment [21, \#112, with the three grids.

We study the turbulent correlations of density with the $z$ component of the velocity, $u_{z}$, in Fig. 3. This correlation is related to gradient diffusion models for subscale turbulence models.

Conventionally, velocity fluctuations are studied using mass weighted averages, $\widetilde{v}=\langle\rho v\rangle / \bar{\rho}$, and as such serve to define the Reynolds stress

$$
R=\langle\widetilde{v v}\rangle-\frac{\langle\widetilde{v} \widetilde{v}\rangle}{\bar{\rho}} .
$$




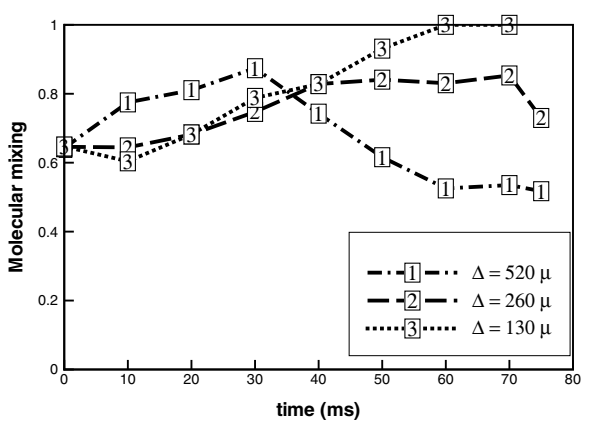

Fig. 2. Plot of the molecular mixing correlation, $\theta$, vs. time for a numerical simulation of the experiment 21 \#112. Three levels of grid refinement are shown: $\Delta x=520,260,130 \mu . \theta$ is evaluated at the mid plane value of $z$, as an average over all of $x, y$.

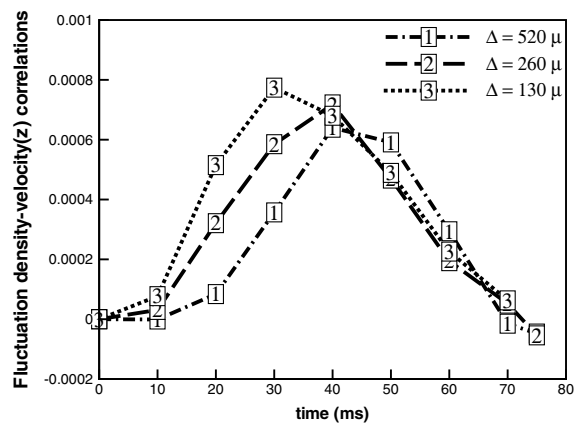

Fig. 3. Plot of $\overline{\rho^{\prime} u_{z}^{\prime}}$ vs. time. Data from the $Z$ midplane, averaged over all of $x, y$.

In Fig. 4 we display the simulated Reynolds stress values for [21] experiment \#112. The convergence properties for $R_{z z}$ appear to be satisfactory (Fig. 4. left). The medium and fine grid display a reasonable level of agreement, while the coarse grid shows a significant discrepancy to the fine grid.

A sensitive comparison is that of $R_{x x}$ to $R_{y y}$, see Fig. 4, right frame. These quantities should be (statistically) identical, so that the solid and dashed curves of the same mesh level family should coincide. This property holds at early but not late time, with the period of agreement increasing under mesh resolution. Moreover, the three curve families should show convergence under mesh refinement, a property which is observed at least up to the time for coincidence of $R_{x x}$ and $R_{y y}$. The difficulty in the convergence of these quantities appears to be related to the inherently small size of the correlations relative to the statistical noise present in their evaluation and to the loss of statistical significance at late time. As the solution progresses, the correlation length increases, an inherent 

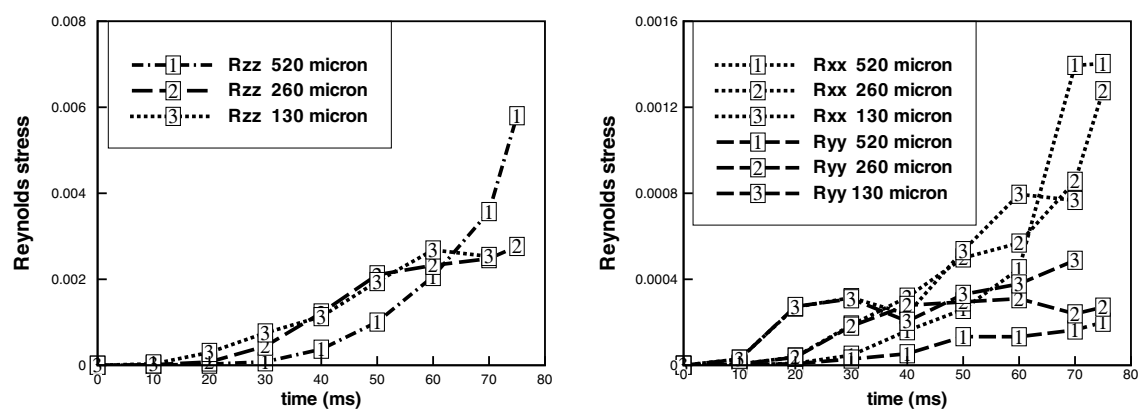

Fig. 4. Plot of Reynolds stress $R_{z z}$ (left) and $R_{x x}, R_{y y}$ (right) vs. time

feature of RT mixing. See the $v_{z}$ gray scale plot at $t=50$ in Fig. 5 , right frame. Thus at late time, the statistical averaging to define $R$ is drawn from a reduced number of independent degrees of freedom, introducing small sample effects into these components of $R$ at late time.

Similar behavior is observed for $R_{x z}$ and $R_{y z}$, see Fig. 5, Due to the rotational symmetry of the statistical formulation, in the case of an infinite $x, y$ domain, these components should be zero, and any non-zero value is a finite size effect in the statistical sampling. There is satisfactory agreement with these two quantities between each other and with zero, up to a time which depends on the mesh. Because the quantities are sensitive to the sign of $v_{z}$ statistically, they have enhanced randomness and decreased convergence properties relative to $R_{x x}$ and $R_{y y}$; they possibly also show small sample size effects at late time.

\subsection{Convergence of PDFs and CDFs}

To define $w^{*}$ convergence, we need to partition the simulation resolution into resources assigned to the conflicting objectives of spatial resolution and statistical resolution. We consider again the midplane $z=$ const and $t=$ const, and partition the $x, y$ plane into supercells. We consider several values for the supercell grid, but show detailed results for an $8 \times 2$ supercell grid. Here the coarsest grid has for each supercell a resolution $9 \times 6$ with a $z$ resolution of a single cell. For the medium and fine grids, the supercell partition is unchanged, but the number of cells in each direction increases by factors of 2 and 4 .

For each supercell, we bin the concentration values into 5 bins, and count the number of values lying in each bin, to obtain a probability. In principle, the number of bins is another parameter in the analysis, variations in which are not explored here. The result of this exercise is an $8 \times 2$ array of PDFs, each represented in the form of a bar graph. The array is a graphical presentation of the Young measure at the fixed $z, t$ value. See Fig. 6. From this array of PDFs, we can observe some level of coherence or continuity in the spatial arrangement of the PDFs, in that the central supercells have a strong heavy fluid concentration, while near the top and bottom, there is more of a mixed cell concentration. 

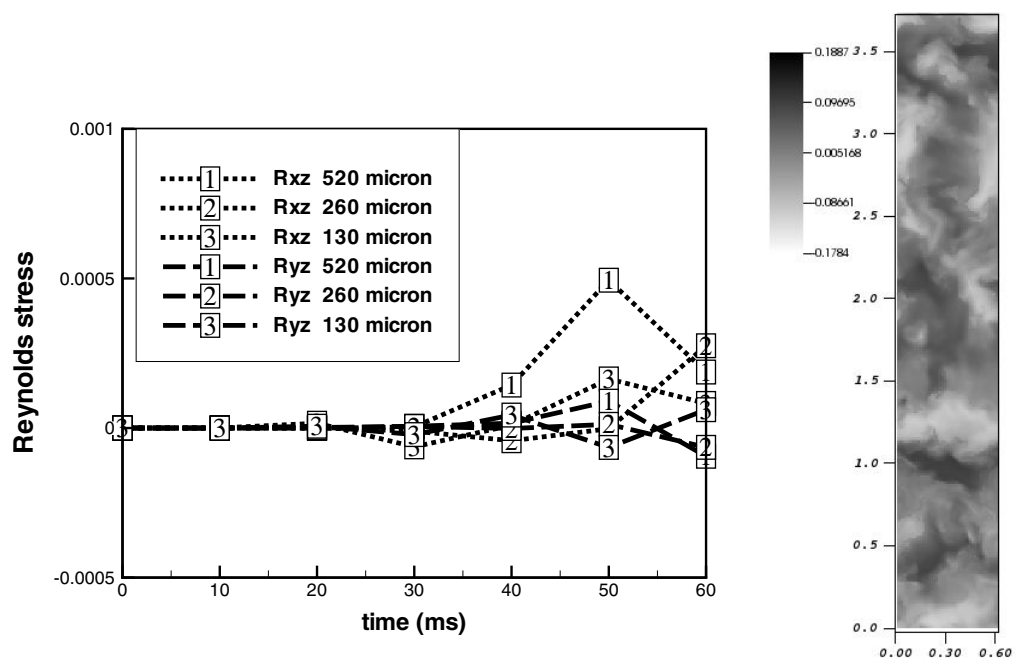

Fig. 5. Left: Plot of Reynolds stress $R_{x z}, R_{y z}$ vs. time. Plotting time is restricted to a maximum of $t=60$ as discussed in the text. Right: Plot of $v_{z}$ (fine grid, $t=50$ ) in the midplane.

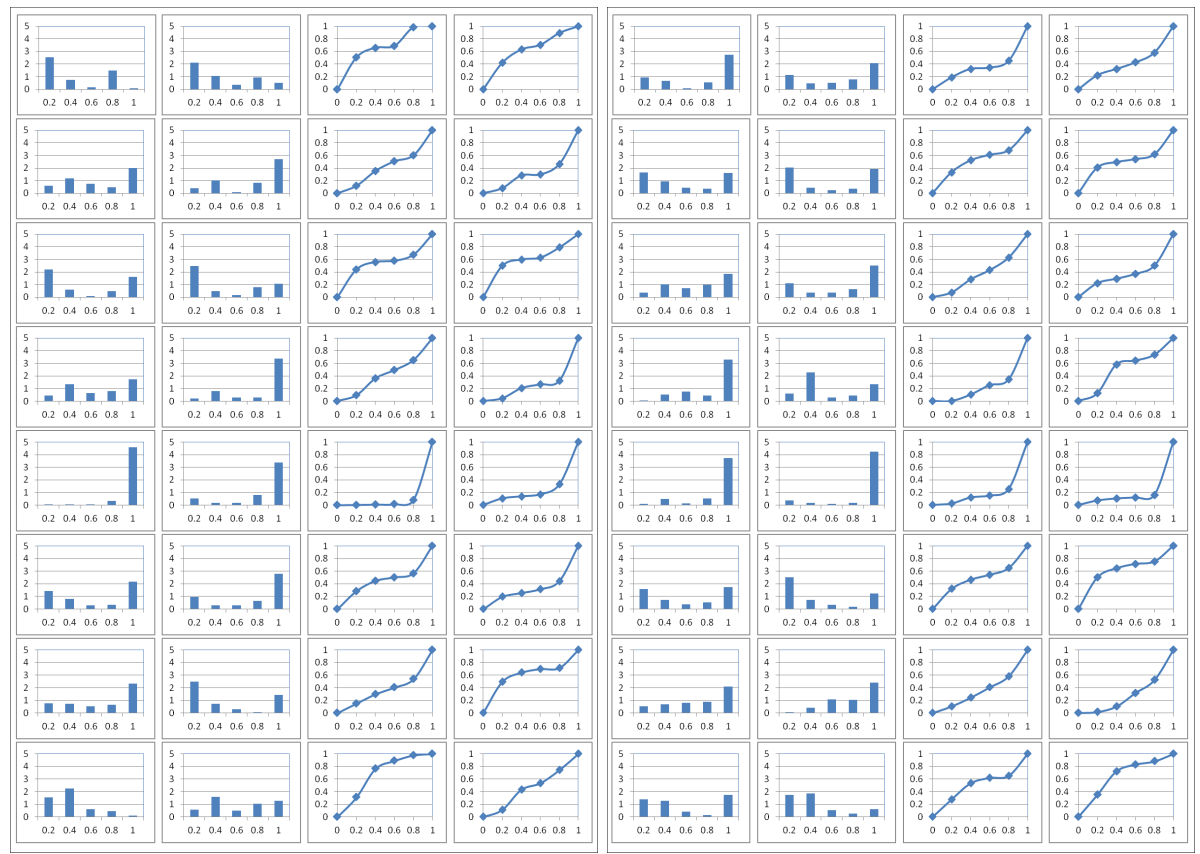

Fig. 6. Spatial array of heavy fluid concentrations at $\mathrm{t}=50$, for $z$ in the midplane, as PDFs (bar graphs) and as CDFs (line graphs), Left: Medium grid. Right: Fine Grid. 

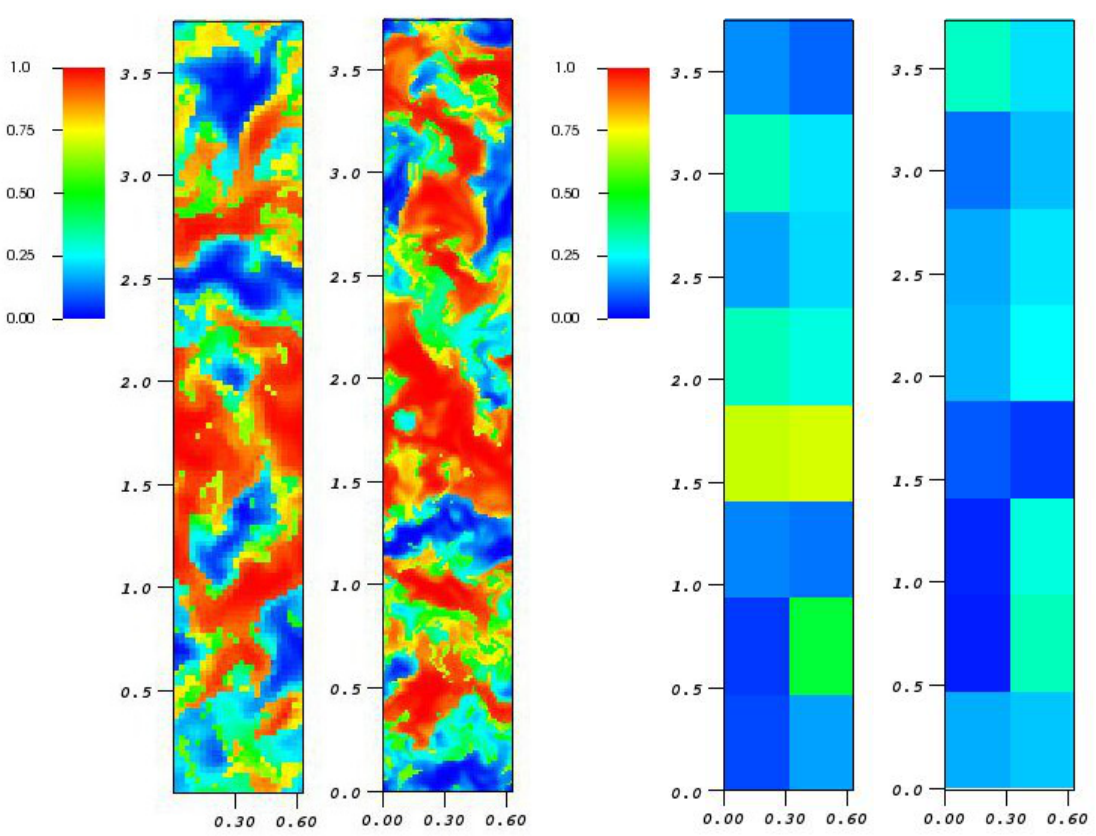

Fig. 7. Left: Plot of heavy fluid concentration at the midplane, $t=50$. Medium grid (left). Fine grid (right). Right: Spatial array of $L_{1}$ norms of CDF mesh differences for heavy fluid concentrations at the midplane. Coarse to fine (left). Medium to fine (right).

Next we study mesh convergence of this $8 \times 2$ array of PDFs and CDFs. At the latest time completed for the fine grid, we compare the PDFs and CDFs on the coarse to fine and medium to fine grids. The comparison is to compute the $L_{1}$ norm of the pairwise differences for each of the $8 \times 2$ PDFs or CDFs. These differences yield an $8 \times 2$ array of norms, i.e. numbers, which is plotted in gray scale in Fig. 7.

The main results of this paper, namely the PDF and CDF convergence properties, are presented in Fig. 7. This data is further simplified by use of global norms. With an $L_{1}$ norm of the differences of the PDFs or CDFs for concentrations in each supercell, we consider both the $L_{1}$ and $L_{\infty}$ norms relative to $x, y$ variables. With the convergence properties thus reduced to a single number, we next explore the consequence of varying the definitions used for convergence. These are (a) the mesh, (b) PDF vs. CDF, (c) $L_{1}$ vs. $L_{\infty}$ for a spatial norm and (d) the size of the supercell used to define the statistical PDF. See Table 3.

We see a convergence trend in all cases under mesh refinement, but useful results for current meshes are limited to CDF convergence. Generally $L_{1}$ norms show better convergence, and generally there is a minimum size for the supercell to obtain useful convergence. Since our convergence properties are documented for the medium grid (through comparison to the fine grid), we can speculate that the errors at the fine grid level would be smaller and that some of the above restrictions might be relaxed in this case. 
Table 3. Summary norm comparison of convergence for heavy fluid concentration PDFs and CDFs at fixed values of $z, t$. In each supercell, an $L_{1}$ norm is applied to the difference of the PDFs or CDFs; this $x, y$ dependent set of norms is measured by an $L_{1}$ or $L_{\infty}$ norm. The larger supercell sizes, the last four columns of the table, cover the entire $y$ domain. In this case, the space-time localization of the PDFs/CDFs are in $x, z, t$ only. We observe convergence for CDFs; while the PDF error is decreasing, further refinement will be needed for usefully converged PDF errors. We see that a coarsening of the supercell resolution (increase of the supercell size) to $18 \times 12$ coarse grid cells per supercell is needed to obtain single digit convergence errors.

\begin{tabular}{|l|c|c|c|c|c|c|}
\hline coarse grid supercell size & \multicolumn{2}{|c|}{$9 \times 6 \times 1$} & \multicolumn{2}{c|}{$18 \times 12 \times 1$} & \multicolumn{2}{c|}{$36 \times 12 \times 1$} \\
mesh comparison & \multicolumn{2}{|c|}{$L_{1}$ norm $L_{\infty}$ norm } & \multicolumn{2}{c|}{$L_{1}$ norm $L_{\infty}$ norm } & \multicolumn{2}{c|}{$L_{1}$ norm $L_{\infty}$ norm } \\
\hline CDFs: coarse to fine & 0.26 & 0.98 & 0.16 & 0.48 & 0.15 & 0.39 \\
CDFs: medium to fine & 0.18 & 0.54 & 0.08 & 0.16 & 0.03 & 0.10 \\
PDFs: coarse to fine & 0.93 & 4.89 & 0.59 & 2.40 & 0.54 & 1.98 \\
PDFs: medium to fine & 0.64 & 2.66 & 0.30 & 0.82 & 0.15 & 0.52 \\
\hline
\end{tabular}

\section{Turbulent Combustion}

Here we explain a primary rationale for our approach to convergence based on fluctuations, PDFs and Young measures. The stochastic convergence to a Young measure is certainly an increase in the complexity of the intellectual formalism in contrast to a more conventional view of convergence to weak solutions.

A simple rationale for the more complicated approach is that pointwise convergence to a weak solution generally fails in turbulent flows. New structures emerge with each new level of mesh refinement and the detailed (pointwise) flow properties are statistically unstable and in fact not observed to converge. Rather, statistical measures of the solutions, of a nature that an experimentalist would call reproducible, are used for convergence studies and these do generally display convergence. Thus we believe that our point of view finds roots in common practices for turbulent study.

In the case of reactive flow (or more generally of a nonlinear process applied to the flow), the stochastic convergence displays its power. Convergence of averages is not usable in a study of nonlinear functions, which require an independent convergence treatment. The LES formulation, moreover, is based on (grid cell or filter) averages. Thus the primitive quantities of an LES simulation cannot be used reliably if a nonlinear process (such as combustion) occurs in the fluid.

The conventional cure for LES turbulent combustion is a model of the flame structure and an assumption that the flame follows a steady state path in concentration-temperature space, with the partially burned state parametrized through a reaction progress variable [18. This assumption leads to a model, called a flamelet model, imposed on the normal turbulent and mixing models. The approach adopted here, in contrast, allows direct computation of the chemistry of a turbulent flame in an LES framework, without the use of (flame 
structure) models. This approach is called finite rate chemistry. Conventionally, finite rate chemistry is possible for DNS only and the extension to LES is a major benefit derived from the stochastic convergence ideas advanced here.

Preliminary results are presented in 9] and will not be reviewed here.

Acknowledgements. This work is supported in part by the Nuclear Energy University Program of the Department of Energy, project NEUP-09-349, Battelle Energy Alliance LLC 00088495 (subaward with DOE as prime sponsor), Leland Stanford Junior University 2175022040367A (subaward with DOE as prime sponsor), Army Research Office W911NF0910306. Computational resources were provided by the Stony Brook Galaxy cluster and the Stony Brook/BNL New York Blue Gene/L IBM machine. This research used resources of the Argonne Leadership Computing Facility at Argonne National Laboratory, which is supported by the Office of Science of the U.S. Department of Energy under contract DE-AC02-06CH11357.

\section{References}

1. Chen, G.Q., Glimm, J.: Kolmogorov's theory of turbulence and inviscid limit of the Navier-Stokes equations in $R^{3}$. Commun. Math. Phys. (2010) (in press)

2. Dimonte, G., Youngs, D.L., Dimits, A., Weber, S., Marinak, M., Wunsch, S., Garsi, C., Robinson, A., Andrews, M., Ramaprabhu, P., Calder, A.C., Fryxell, B., Bielle, J., Dursi, L., MacNiece, P., Olson, K., Ricker, P., Rosner, R., Timmes, F., Tubo, H., Young, Y.N., Zingale, M.: A comparative study of the turbulent RayleighTaylor instability using high-resolution three-dimensional numerical simulations: The alpha-group collaboration. Phys. Fluids 16, 1668-1693 (2004)

3. George, E., Glimm, J., Li, X.L., Li, Y.H., Liu, X.F.: The influence of scale-breaking phenomena on turbulent mixing rates. Phys. Rev. E 73, 016304 (2006)

4. Glimm, J., Sharp, D.H., Kaman, T., Lim, H.: New directions for Rayleigh-Taylor mixing. Philosophical Transactions of The Royal Society A: Turbulent Mixing and Beyond (2011), submitted for publication; Los Alamos National Laboratory National Laboratory preprint LA UR 11-00423. Stony Brook University preprint number SUNYSB-AMS-11-01

5. Kaman, T., Glimm, J., Sharp, D.H.: Initial conditions for turbulent mixing simulations. Condensed Matter Physics 13, 43401 (2010), Stony Brook University Preprint number SUNYSB-AMS-10-03 and Los Alamos National Laboratory Preprint number LA-UR 10-03424

6. Kaman, T., Glimm, J., Sharp, D.H.: Uncertainty quantification for turbulent mixing simulation. In: ASTRONUM, Astronomical Society of the Pacific Conference Series (2010), Stony Brook University Preprint number SUNYSB-AMS-10-04. Los Alamos National Laboratory preprint LA-UR 11-00422 (submitted)

7. Kaman, T., Lim, H., Yu, Y., Wang, D., Hu, Y., Kim, J.D., Li, Y., Wu, L., Glimm, J., Jiao, X., Li, X.L., Samulyak, R.: A numerical method for the simulation of turbulent mixing and its basis in mathematical theory. In: Lecture Notes on Numerical Methods for Hyperbolic Equations: Theory and Applications: Short Course Book, pp. 105-129. CRC/Balkema, London (2011), Stony Brook University Preprint number SUNYSB-AMS-11-02 
8. Lim, H., Iwerks, J., Glimm, J., Sharp, D.H.: Nonideal Rayleigh-Taylor mixing. PNAS 107(29), 12786-12792 (2010), Stony Brook Preprint SUNYSB-AMS-09-05 and Los Alamos National Laboratory preprint number LA-UR 09-06333

9. Lim, H., Kaman, T., Yu, Y., Mahadeo, V., Xu, Y., Zhang, H., Glimm, J., Dutta, S., Sharp, D.H., Plohr, B.: A mathematical theory for LES convergence. Acta Mathematica Scientia (2011) (submitted for publication), Stony Brook Preprint SUNYSB-AMS-11-07 and Los Alamos National Laboratory preprint number LAUR 11-05862

10. Lim, H., Yu, Y., Glimm, J., Li, X.L., Sharp, D.H.: Subgrid models in turbulent mixing. In: Astronomical Society of the Pacific Conference Series, vol. 406, p. 42 (2008), Stony Brook Preprint SUNYSB-AMS-09-01 and Los Alamos National Laboratory Preprint LA-UR 08-05999

11. Lim, H., Yu, Y., Glimm, J., Li, X.L., Sharp, D.H.: Subgrid models for mass and thermal diffusion in turbulent mixing. Physica Scripta T142, 014062 (2010), Stony Brook Preprint SUNYSB-AMS-08-07 and Los Alamos National Laboratory Preprint LA-UR 08-07725

12. Lim, H., Yu, Y., Glimm, J., Sharp, D.H.: Nearly discontinuous chaotic mixing. High Energy Density Physics 6, 223-226 (2010), Stony Brook University Preprint SUNYSB-AMS-09-02 and Los Alamos National Laboratory preprint number LAUR-09-01364

13. Liu, X.F., George, E., Bo, W., Glimm, J.: Turbulent mixing with physical mass diffusion. Phys. Rev. E 73, 056301 (2006)

14. Moin, P., Squires, K., Cabot, W., Lee, S.: A dynamic subgrid-scale model for compressible turbulence and scalar transport. Phys. Fluids A3, 2746-2757 (1991)

15. Mueschke, N., Schilling, O.: Investigation of Rayleigh-Taylor turbulence and mixing using direct numerical simulation with experimentally measured initial conditions.

i. Comparison to experimental data. Physics of Fluids 21, 014106-1-014106-19 (2009)

16. Mueschke, N., Schilling, O.: Investigation of Rayleigh-Taylor turbulence and mixing using direct numerical simulation with experimentally measured initial conditions. ii. Dynamics of transitional flow and mixing statistics. Physics of Fluids 21, 0141071-014107-16 (2009)

17. Mueschke, N.J.: Experimental and numerical study of molecular mixing dynamics in Rayleigh-Taylor unstable flows. Ph.D. thesis, Texas A and M University (2008)

18. Pitsch, H.: Large-eddy simulation of turbulent combustion. Annual Rev. Fluid Mech. 38, 453-482 (2006)

19. Ramaprabhu, P., Andrews, M.: Experimental investigation of Rayleigh-Taylor mixing at small atwood numbers. J. Fluid Mech. 502, 233-271 (2004)

20. Read, K.I.: Experimental investigation of turbulent mixing by Rayleigh-Taylor instability. Physica D 12, 45-58 (1984)

21. Smeeton, V.S., Youngs, D.L.: Experimental investigation of turbulent mixing by Rayleigh-Taylor instability (part 3). AWE Report Number 0 35/87 (1987) 


\section{Discussion}

Speaker: James Glimm

Bill Oberkampf: You have presented several new ideas in both V\&V as well as UQ that are very innovative. I have two questions. What advantages do you see in considering temporal and statistical convergence of PDF's of quantities of interest, as opposed to convergence of time averaged quantities at a point?

James Glimm: See Sec. 5 .

Bill Oberkampf: When you examine mesh and temporal convergence in LES simulations you are merging changing sub-grid scales (resulting in changes in the math model) and changing numerical solution error. Since these are very different sources of uncertainty, what are your ideas for separating these uncertainties?

James Glimm: This is an excellent and deep question, whose answer is context dependent. For the case of turbulence models, we will address this issue in a separate publication (manuscript in preparation). Briefly, and for turbulent mixing, the SGS turbulent terms to be added to the Navier Stokes equations have a formulation in terms of gradients of primitive solution variables, if the dynamic subgrid models [14] are used. For this closure, convergence of the turbulence SGS model terms is a numerical analysis issue, as is already defined from the perspective of physics and modeling. Verification and validation of these models (a much studied topic) should be addressed in each separate simulation or flow regime. See for example, Sec. 4 . 